\title{
Freiwilliger Verzicht auf Essen und Trinken - zur ethischen Lagebestimmung eines ambivalenten Begriffs
}

\author{
Philipp Starke
}

Eingegangen: 27. August 2019 / Angenommen: 16. Dezember 2019 / Online publiziert: 9. März 2020

(C) Der/die Autor(en) 2020

Zusammenfassung Wie ist der Freiwillige Verzicht auf Essen und Trinken und eine medizinische Begleitung dabei ethisch zu bewerten? Die ethische Bewertung des Freiwilligen Verzichts auf Essen und Trinken stellt Patienten und Angehörige, aber auch begleitende Ärzte und Pflegende vor erhebliche Schwierigkeiten. Basierend auf Ergebnissen eigener qualitativer Interviews mit Personen nach dem FVET ihrer Angehörigen legt dieser Artikel die bestehende Unklarheit und inhärente ethische Ambivalenz des Begriffs FVET frei, stellt aber in der Unterscheidung von FVET-Fällen - mit bzw. ohne terminale Erkrankung - einen Weg zur Lösung der Frage nach der ethischen Bewertung des „Sterbefastens“ und seiner medizinischen Begleitung vor: Ethisch wird der Freiwillige Verzicht auf Essen und Trinken und seine medizinische Begleitung erst in der Analyse von Situation und Intention der Handelnden beurteilbar. Ausgehend vom Konzept eines frei gewollten Verzichts auf Essen und Trinken bei einsichts- und einwilligungsfähigen Personen mit dem Bewusstsein, damit ihr Leben zu verkürzen oder zu beenden, wird mit der Philosophie Thomas von Aquins argumentiert, dass eine ethische Bewertung die Begleitumstände und beabsichtigten Ziele miteinschließen muss, ebenso wie die Auswirkungen auf Angehörige und die Implikationen von und für ein Behandlungsteam.

Schlüsselwörter Freiwilliger Verzicht auf Essen und Trinken · FVET · Freiwilliger Verzicht auf Nahrung und Flüssigkeit · FVNF · VSED · Sterbefasten · Qualitative Forschung · Thomas von Aquin

Die vorliegende Arbeit wurde mit dem Nachwuchspreis 2019 der Akademie für Ethik in der Medizin e. V. ausgezeichnet.

P. Starke $(\bowtie)$

Institut für Ethik, Geschichte und Theorie der Medizin, Ludwig-Maximilians-Universität München, Lessingstraße 2, 80336 München, Deutschland

E-Mail: philipp.starke@campus.Imu.de 


\title{
Voluntary stopping of eating and drinking. On tackling its ethical ambivalence
}

\begin{abstract}
Definition of the problem An ethical evaluation of voluntary stopping of eating and drinking (VSED) continues to be challenging not only for patients and proxies, but also for accompanying physicians and nurses. This article intends to clarify the existing ambiguity and ethical ambivalence often encountered when discussing VSED.

Arguments Informed by qualitative research with proxies of VSED persons, the distinction of individuals with and without terminal disease is introduced as a gamechanging factor when tackling VSED in the ethical field. Setting the precondition of an autonomous patient with full decision-making capacity and basing on Thomas Aquinas' fonts of morality, to look at intention and situation helps to ethically evaluate VSED and its medical support.

Conclusion Focusing on the primary intentions of terminally ill patients-moderating, not intentionally causing death-VSED appears ethically acceptable. Consequently, support by health care professionals should be provided, valuable both for the person concerned as well as her proxies. In cases of non-terminally ill persons, accompanying VSED needs to be answered in a broader context: specifically, of how to address the wish to end life by a person possessing full decision-making capacity.
\end{abstract}

Keywords Voluntary stopping of eating and drinking · VSED - Qualitative research · Thomas Aquinas

\section{Einleitung}

Der Freiwillige Verzicht auf Essen und Trinken (FVET), „Sterbefasten“ oder engl. Voluntary Stopping of Eating and Drinking (VSED) erfährt aktuell zunehmend Aufmerksamkeit (Coors et al. 2019). Über Gründe und Motive dieses gestiegenen Interesses an einer uralten Form des Sterbens - erste Erwähnungen datieren in die Antike zurück ${ }^{1}$ - lässt sich spekulieren. Das Interesse erscheint in Deutschland aber hinreichend plausibel vor dem Hintergrund einer demografisch alternden Gesellschaft, zunehmend ausgefeilten aber selten nebenwirkungsarmen Therapieoptionen und letztlich als Folge des Verbots der geschäftsmäßigen Förderung der Selbsttötung (Rosenau 2016). Der FVET scheint für nicht wenige Betroffene eine gangbare Alternative dazu darzustellen, eine Art „Ausweg am Lebensende“ (Chabot und Walther 2015).

\footnotetext{
${ }^{1}$ So beispielsweise über den griechischen Philosophen und „Hungerselbstmörder“ Hegesias (s. Cicero 1998, Tusc. I,84) oder die Praxis des sallekhana des Jainismus (Freiberger und Kleine 2015, S. 452).
} 
Wie aber ist der FVET und vor allem die professionelle Begleitung durch Ärzte ${ }^{2}$ und Pflegende ethisch zu werten? Dieser Leitfrage möchte sich der folgende Artikel stellen, in Form einer philosophischen Auseinandersetzung unter individual- und sozialethischen Gesichtspunkten. Im Unterschied zu bisherigen Beiträgen basieren die Überlegungen auf den Ergebnissen eigener qualitativer Forschung aus Angehörigeninterviews in Deutschland und der Schweiz. Diese bieten zwar keine ethischnormative Aussagekraft, wohl aber eine Orientierung zur Lebenswelt, den Motiven und Erfahrungen der beteiligten Personen - und so Anhaltspunkte für nötige Unterscheidungen bei der ethischen Bewertung des FVET und seiner professionellen Begleitung.

\section{Was ist „FVET“ - und was nicht?}

Aktuell existieren mehrere Begriffe zum Thema, die meist parallel oder synonym verwendet werden: Ausgehend von engl. Voluntary Refusal of Food and Fluids (VRFF) (Bernat et al. 1993; Quill et al. 2000; Ganzini et al. 2003) und Voluntary Stopping of Eating and Drinking (VSED) (Quill et al. 1997) haben sich im deutschsprachigen Raum wörtliche Übersetzungen entwickelt: der Freiwillige Verzicht auf Nahrung und Flüssigkeit (FVNF) (Hoekstra et al. 2015; Chabot und Walther 2015; Bickhardt und Hanke 2014) und der Freiwillige Verzicht auf Essen und Trinken (FVET) (Jox et al. 2017). Die semantische Entwicklung weist auf zwei wichtige Aspekte hin.

Erstens lassen $F V N F$ bzw. VRFF offen, ob eine Person sich selbstständig ernähren oder nur mit technischer oder pflegerischer Hilfe ernährt werden kann: Somit umfassen sie auch die Angewiesenheit auf künstliche Ernährung oder darauf, gefüttert zu werden (Birnbacher und Walther 2019). Nahrung und Flüssigkeit bilden semantisch Oberbegriffe, während Essen und Trinken eine Selbstständigkeit der natürlichen, menschlichen Nahrungsaufnahme implizieren. Diese wünschenswerte Differenzierung von FVNF und FVET hat sich bisher noch nicht überall durchgesetzt.

Zweitens nuanciert der Übergang des voluntary refusal zum voluntary stopping eine Veränderung hin zu einer überlegten, rationalen Entscheidung, auf Deutsch vielleicht am ehesten wiedergegeben im Unterschied von Verweigerung zu Verzicht. Diese Präzisierung weist auf den wichtigsten Aspekt des Konzeptes von FVET hin: die ihm zugrundeliegende wohlinformierte und autonomiebasierte - kurz: freie Entscheidung einer Person.

Chabot und Walther prägten zusätzlich den Begriff „Sterbefasten“ (Chabot und Walther 2015), in Anlehnung an das ndl. sterfwerk (Chabot 2001). Damit wird weniger präzise der Weg verdeutlicht, eindeutig aber das Ziel: Im Gegensatz zu den anderen Begriffen denkt „Sterbefasten“ die Handlung vom Ende und der Intention der Handelnden aus - fokussiert also auf die selbstverfügte Verursachung oder Inkaufnahme des Todes. Ausmaß, Art und Situation des Verzichts bleiben damit offen und ob die Lesart Fasten im Sterben oder Fasten zum Sterben gemeint sein soll.

\footnotetext{
${ }^{2}$ Die Verwendung des generischen Maskulinums erfolgt ausschließlich zur Erleichterung des Leseflusses. Es sind jeweils alle Geschlechtsbezeichnungen mitgemeint.
} 
So verschleiert der Begriff „Sterbefasten“ zunächst eine ethische Bewertung und führt mit dem Fokus des beabsichtigten Lebensendes oft zum Kurzschluss, jedes „Sterbefasten“ sei ein Suizid ${ }^{3}$ (Wikipedia ${ }^{4}$ ). Fasten ist zudem primär spirituell oder medizinisch-therapeutisch konnotiert, was den Begriff angreifbar und für manche unannehmbar macht (Kittelberger 2018; Klein 2018). Trotzdem hat sich ,Sterbefasten " außerhalb des akademischen Diskurses fest etabliert - es bleibt zu hoffen, dass eine semantische Konkretisierung als Synonym zum FVET nur eine Frage der Zeit ist.

Definitorisch umschließt der Freiwillige Verzicht auf Essen und Trinken also das willentliche, in freier, wohlinformierter und selbstbestimmter Entscheidung beschlossene Unterlassen oder möglichst große Verringern von Essen und Trinken im Bewusstsein, damit das eigene Leben zu verkürzen oder das Lebensende herbeizuführen.

\section{Qualitative Forschung zum FVET}

\section{Methodik}

Für die Ethik als Disziplin der praktischen Philosophie ist ein profundes Verständnis des Untersuchungsgegenstands notwendig, hier der Lebenswelt der Beteiligten. Zu diesem Zweck habe ich mit 18 Personen, die den FVET eines nahestehenden Angehörigen erlebt haben, semi-strukturierte, qualitative Interviews durchgeführt. Die Gespräche fanden dabei in Deutschland (14) und der Schweiz (4) auf Deutsch oder Französisch statt, jeweils mindestens drei Monate nach dem Tod des Angehörigen. Im Rahmen eines Mixed-Methods-Design wurde jedes Gespräch von einem numerischen Fragebogen ergänzt. Diese Ergebnisse können auf Grund der kleinen Teilnehmerzahl die qualitativen Erkenntnisse aber allenfalls konkretisieren. Die Interviews wurden wörtlich transkribiert und mittels qualitativer Inhaltsanalyse nach Mayring ausgewertet (Mayring 2015). Wichtige Gesprächsthemen der Interviews waren Prozess und Situation der Entscheidungsfindung, die Phase des Verzichts selbst sowie das Erleben der Trauer und eine retrospektive Bewertung durch die Angehörigen. Die Rekrutierung erfolgte vor allem über stationäre und ambulante Palliativ- und Hospizdienste, Alten- und Pflegeheime sowie über persönlichen Kontakt. Das internationale Design wurde insbesondere zur Evaluierung des je geltenden Rechtsrahmens gewählt. Eingeschlossen wurden nur volljährige Personen, deren schriftliches Einverständnis zur Teilnahme vorlag und die in einer engen Beziehung zu ihrem Angehörigen auch während dessen FVET gestanden hatten. Die Studie wurde von der zuständigen Ethikkommission geprüft und bewilligt.

\footnotetext{
3 Ob FVET und „Sterbefasten“ philosophisch und/oder rechtlich Suizide sind, bleibt umstritten (S. bes. Birnbacher 2019, 2015; Neitzke 2019).

4 www.wikipedia.de: Sterbefasten. https://de.wikipedia.org/wiki/Sterbefasten. Zugegriffen: 26. Okt. 2019.
} 


\section{Ergebnisse}

\section{Der Prozess der Entscheidungsfindung zum FVET}

Für einen FVET muss die Einsichts- und Entscheidungsfähigkeit der Person vollumfänglich gewährleistet sein. Die Entscheidungsfindung zum FVET findet dann vereinfacht in zwei voneinander abgrenzbaren Situationen statt.

\section{a) Am Ende einer lebensbedrohenden Erkrankung}

Eine typische Entscheidungssituation von Patienten zum FVET ist das Spätstadium einer unheilbaren Krankheit - insbesondere onkologischer oder degenerativer Natur. Die Patienten sehen sich an einem Punkt, wo keine reale Hoffnung auf Heilung mehr besteht und die Lebensqualität von der Erkrankung zunehmend eingeschränkt wird: „Und wo wir dann einen Arzt gefunden hatten, wo dann eine Methode angeschlagen hatte, war sie schon so schwach, war der Körper schon so kaputt, dass sie irgendwann mal zu mir gesagt hat: ,[Name des Ehemanns], ich will nicht mehr. Ich weiß nicht, wie das weitergehen soll und das macht in diesem Leben einfach keinen Sinn mehr in diesem Körper'. Und da hat sie einfach gesagt: „Das Sinnvollste ist einfach nichts mehr zu essen und zu trinken. “ (OJ) Die Fülle von Symptomen - gerade bei onkologischen Patienten - wird als körperlich und psychisch schwer belastend erlebt und motiviert oft die Entscheidung. Bei neuro-degenerativen Erkrankungen betonen Angehörige zudem das nahende Lebensende und das Erleben des Fortschreitens der Erkrankung. In solchen als ausweglos erlebten Lagen ziehen Angehörige, aber auch Ärzte, nicht selten eine Parallele zu Situationen, für die der Gesetzgeber in der Schweiz den medizinisch assistierten Suizid zulässt (vgl. Art. 115 Schweizerisches Strafgesetzbuch StGB, Art. 27d Loi de la Santé Publique, Vaud): „Und dann war es klar, er muss operiert werden, um diese Schmerzen da, diese Metastasen in der Wirbelsäule wegzukriegen. [Aber dann war] die Querschnittslähmung da. Und das war für ihn der Punkt, dass er mich aus dem Krankenhaus angerufen hat und gesagt hat: ,Fahr mich nach Zürich!' [...] Und dann kam die Frau Dr. [Palliativmedizinerin] von der Palliativstation und hat ihm die Hoffnung gemacht: ,Eh, Sie müssen nicht nach Zürich fahren, es gibt auch hier in Deutschland die Möglichkeit [erg. des FVET]. ' Und dann ist er echt wieder aufgeblüht, weil er die Chance gesehen hat - wunderbar - hat sich das genau beschreiben lassen. Sterbefasten kannte er vorher nicht, ich auch nicht. " (XM).

Bei schwer erkrankten Patienten verliert Essen häufig an Bedeutung, so dass ein fließender Übergang zwischen dem FVET und dem Verlauf der Krankheit möglich ist: „Also er hat auch aufgehört mit dem Essen, weil es ihm auch kaum mehr geschmeckt hat. [...] Und hat gesagt: ,Na dann lassen wir das doch.' Gut. Und dann hat er getrunken, das hat er noch, aber viel zu wenig. “ (FZ) Der Entschluss zum FVET fällt oft in diesem unscharfen Übergang: „Es hat ihr nichts mehr geschmeckt zum einen - sie hat es auch nicht mehr gut vertragen - aber ihre Grundmeinung war einfach: Ich höre das einfach auf, dann kann ich in Ruhe sterben und dann geht es auch schneller. " (SR).

Der FVET wird von diesen Patienten und Angehörigen zum Ende des Krankheitsweges - also nach Abwägung und meist nach Ausschöpfung aller kurativen Versuche 
- dann als eine letzte Option gesehen. „Aber wir haben bis zum letzten Moment auch die Therapiemöglichkeiten in Erwägung gezogen und uns das sehr genau angeguckt. Aber da war einfach nichts mehr dabei, was ihm hätte helfen können." (FZ) Die Entscheidung zum FVET wird bei dieser Gruppe letztlich wenig oder gar nicht von einem akuten Sterbewunsch motiviert, sondern vom Wunsch, ihr absehbares Sterben durch FVET beeinflussen zu können - und besonders zu Hause zu sterben. „Ja [...], weil wir überlegt hatten, wie es für ihn am ,schönsten' am normalsten sein könnte. Und zu Hause ist normal. [...] In [der Uniklinik] war er gut betreut, das ist es nicht, aber es ist ein Krankenhaus und er wollte immer nach Hause. “ (FZ).

Zusammenfassend wählt diese erste Gruppe von Personen den FVET also als eine „letzte Option“ am Ende eines langen, medizinisch begleiteten Wegs. Der Übergang von den Symptomen der Erkrankung zum FVET kann dabei fließend sein. Die Patienten sehen den FVET vor allem als Chance, weiteres Leid zu vermeiden oder zu verkürzen, das Sterben zu beschleunigen sowie auf den Sterbeort und die Umstände Einfluss nehmen zu können.

b) Höherer Sterbe- als Behandlungswunsch: FVET als Freitod

Die zweite Gruppe von Personen entscheidet sich zum FVET nicht auf Grund palliativer Überlegungen und in Absehbarkeit des Lebensendes, sondern aus dem primären Wunsch heraus, nicht mehr weiterleben zu wollen. Dieser Sterbewunsch wird oftmals mit schwindender Lebensqualität durch eine chronische Erkrankung oder hohes Alter begründet und besteht meist schon für mindestens einige Monate. Den Auslöser für die Entscheidung zum FVET kann dann eine Verschlechterung im Rahmen einer chronischen Erkrankung oder ein Klinikaufenthalt darstellen: „Mobilität und überhaupt Aktivität [war] für ihn lebensnotwendig. [...] Und dann aber hatte er [..] einen Ermüdungsbruch in der Wirbelsäule. [...] Aber da hat ihm die Dr. [Palliativmedizinerin] dann auch gesagt: ,Ich kann Sie schmerzfrei kriegen - würden sie dann weiterleben wollen?' Und er hat gesagt: ,Nein, also wenn ich dann nur hier an dieser Morphiumpumpe im Bett liegen muss, dann ist das für mich nicht lebenswert. “ (DZ) Für ihre Entscheidung ist hier ein Vorrang anderer Motive vor rein medizinischen grundlegend: Obwohl eine Erkrankung nicht lebensbedrohlich ist, wünschen sich diese Personen eher ein rasches Lebensende, da sie eine (weitere) Minderung ihrer Lebensqualität nicht tolerieren möchten.

Dieser Kreis umschließt ebenso Personen, die mit kaum einer oder ohne eine Grunderkrankung leben, wie die hochbetagte Frau H., die zur erfolgreichen Therapie (!) eines verschleppten Harnweginfekts kurzzeitig stationär aufgenommen worden war: „PS: ,Hat Sie Ihnen einen Hauptgrund genannt, weswegen sie mit dem Essen und Trinken aufhören wollte?' Herr H.: ,Sie wollte nicht mehr - wollte ganz klar nicht mehr leben. Das war es, was sie gesagt hat und ich glaube, dass ihr auch klar war, dass wieder nach Hause zu kommen schwierig sein würde, dass das zumindest einen Verlust an Selbstständigkeit bedeuten würde. Und für meine Mutter [...] war das etwas Unmögliches: Verlust an Selbstständigkeit, das war für sie nicht vorstellbar. "(UH) Die Entscheidungsgründe zum FVET können so auch unabhängig von Erkrankungen sein und entziehen sich damit einer direkten Behandlungsmöglichkeit. Gelegentlich entschieden sich Angehörige aber auch vollkommen außerhalb eines medizinischen Kontextes für den FVET, wie die ebenfalls hochbetagte Frau O in der 
Schweiz: „Meine Großmutter war eine Dame von 92 Jahren. Über Jahre hatte sie mir gesagt, dass sie gern sterben würde. [...] Sie war seit achtzehn Jahren verwitwet und war eine sehr selbstständige Frau, ging Tanzen, war voller Leben [...]. Sie hatte ihren Mann verloren, war aber zugleich immer von Familie umgeben. [...] Ich würde also sagen, sie war gut umsorgt, aber sie wollte sterben. Sie hatte einfach genug. Und weil sie wusste, dass ich Sterbebegleiterin bei [einer Suizidhilfe-Organisation in der Schweiz] bin, hat sie mir immer wieder gesagt: Aber Du könntest mir doch helfen! Und ich habe gesagt: Nein, dazu habe ich nicht das Recht. - Und das Paradoxe ist, ich habe sie ja gefragt: ,Aber warum willst Du denn sterben? Dir geht's doch gut! ‘ Ich würde sagen - sie hatte ja keine Schmerzen - es war einfach eine Lebensmüdigkeit. Sie hatte einfach keine Lust mehr zu leben. " (RO) Die Situationen und Motive, die zum FVET führen, müssen damit als weiter gefasst verstanden werden, als allein ,aufgrund unerträglichen anhaltenden Leidens“ (Radbruch et al. 2019) - der Entschluss zum FVET wird in seltenen Fällen allein auf ein hohes Alter und eine „Lebenssattheit“ zurückgeführt.

Zusammenfassend verbindet diese Gruppe der Wunsch, nicht mehr leben zu wollen: Die einzelnen Situationen variieren dabei und können, müssen aber nicht, von einer Erkrankung bestimmt sein. Eine terminale Erkrankung liegt hier nicht vor. Die Motive reichen hier somit durchaus in ein Spektrum, das der Begriff Freitod bezeichnet, also einen im Vollbesitz geistiger Kräfte überlegt gefassten Entschluss der Selbsttötung. ${ }^{5}$

\section{Verzichtphase}

Häufig sind Patienten, besonders terminal Erkrankte, bereits in eine palliativmedizinische Versorgung eingebunden, die unabhängig vom FVET selbstverständlich erhalten bleiben sollte. In beiden Varianten des FVET haben jedoch zwei Aspekte einer ärztlichen Begleitung während der Verzichtsphase ethische Relevanz: die Symptomkontrolle und ihre Auswirkungen sowie die Begleitung von Angehörigen.

Alle Angehörigen berichten von einer spezifischen Symptomatik des FVET: ein sehr starkes Durstgefühl und starke Mundtrockenheit, gleichzeitig aber kaum ein Erleben von Hunger. Dazu treten besonders Schwäche und Müdigkeit, seltener Schlafstörungen und Verwirrtheitszustände auf. Für diese Symptome stehen Therapieansätze zur Verfügung, wobei der professionellen Mundpflege zur Linderung des Durstgefühls und dem Schmerzmanagement eine besondere Rolle zukommt oftmals für Schmerzen, die eine Erkrankung, nicht der Verzicht selbst verursacht.

Wichtig für die ethische Bewertung der Begleitung beim FVET ist, dass die Zusicherung jener für Personen eine Grundvoraussetzung zum FVET sein kann. Ohne professionelle Hilfe kommt ein FVET für manche nicht in Frage: „PS: ,Hat das einen Unterschied gemacht, ob er medizinische Hilfe da bekommen hat, also die-

\footnotetext{
5 Alternativbegriffe wie Selbstmord oder Suizid vermeide ich hier, da sie spezifische Konnotationen (z. B. Sünde) tragen oder oft einen psychiatrisch-klinischen Kontext implizieren - beides deckt sich nicht mit dem FVET. Freitod hingegen gibt m. E. am ehesten den freien Entschluss wieder; ein Euphemisieren des FVET im Begriff Freitod ist nicht intendiert, er ist dazu stets kursiv gesetzt. Zur Begriffsklärung vgl. (Jaspers 1956, S. 300f.).
} 
se Zusicherung von der Frau Dr. [Palliativmedizinerin] sich da auch zu kümmern?' XM: ,Nur. Das war's, absolut. Er war tief verzweifelt, was mache ich jetzt? [...] Was macht auch der Krebs mit mir? Und dann hat sie eben diese Möglichkeit [FVET] genannt und gesagt: Wir können das auch hier machen. " (XM) Für die Entscheidung mancher Patienten ist die Verfügbarkeit palliativmedizinischer Begleitung und adäquater Symptomkontrolle des FVET somit sehr relevant. Damit steht ein Behandlungsteam hier in einer besonderen Verantwortung.

Das Lindern von Symptomen hat zudem Einfluss auf die psychische Konstitution von Patienten. „Das wichtigste ist da eigentlich, dass man mit dem Palliativteam, dass die einem eben Sicherheit geben und dass man sich dann - also für uns war das so - dass man sich auf was anderes konzentrieren kann [...], die Zeit, die man hat, noch so gestalten kann - also so wie man das, wie es einem möglich ist, und wie man das gerne hätte. [...] Das war halt für uns eine Hilfe. " (RE) Gelingen oder Versagen einer palliativmedizinischen Begleitung beim FVET trifft somit nicht nur den Patienten körperlich. Es bestimmt auch, ob und wie er sein Lebensende mit seinen Angehörigen verbringen kann.

Eine ganzheitliche Palliativmedizin hat die Betreuung der Angehörigen auch mit im Blick. Dies ist beim FVET nicht anders und alle Angehörigen schätzen die Begleitung als auch für sie selbst sehr hilfreich ein. „PS: ,Hatten Sie da auch Zeit, mit den Palliativmedizinern, mit der Frau Dr. [Palliativmedizinerin] zu sprechen? Also haben die sich auch ein bisschen um Sie als Angehörige gekümmert?’ VQ: ,Ja. Also vor allem als sie das letzte Mal da war. [...] Ja, das war sehr gut. Das hat es leichter gemacht." "(VQ).

Für eine ethische Beurteilung ist festzuhalten, dass ärztliche Begleitung beim FVET Leiden für den Patienten und seine Angehörigen erheblich verringern, ja eine enorme Hilfe sein kann. Da für manche Patienten ein FVET nur mit dieser Begleitung denkbar ist, sollten sich Entscheidungsträger ihrer Verantwortung bei Zu- bzw. Absage von Unterstützung bewusst sein: Ihre Entscheidung schränkt den Handlungs- und Entscheidungsraum von Patienten möglicherweise stark ein. Die ethische Bewertung der ärztlichen Begleitung von der des FVET selbst sollte daher auch nicht vollständig getrennt werden (Birnbacher 2019; Jox et al. 2017).

\section{Trauerphase}

Die Trauer der Angehörigen nach einem FVET variiert deutlich. Den Verlust der verstorbenen Person, meist nach einer langen Beziehung, schildern Angehörige als für die Trauer wichtiger als den FVET selbst. Bei manchen Angehörigen prägte der FVET den Trauerverlauf mit, dies ebenfalls entlang der Unterscheidung, ob eine terminale Erkrankung vorlag: Gefragt auf einer Skala von null bis zehn nach dem Grad ihrer Zustimmung zur Aussage, der FVET des Angehörigen habe ihr Trauern erschwert, wiesen alle Angehörigen von Patienten mit terminaler Erkrankung dieser Aussage den Wert null zu (,0= stimme gar nicht $\left.\mathrm{zu}^{\prime}\right)$.

Wenn keine terminale Krankheit vorlag, konnte ein FVET die Trauer beeinflussen: „PS: ,Haben Sie das Gefühl, dass dieses Sterbefasten als Sterbefasten die Trauer um Ihre Schwester noch einmal anders beeinflusst hat?' RY: ,Es ist halt so, dass man sagt: man hat zu wenig getan. Das ist ein Vorwurf, der immer wieder kommt. 
Was wäre gewesen, wenn ich mich mehr um sie gekümmert hätte am Schluss? Und warum ich es nicht getan habe. Das ... Das ist sicher da. [...] Und dass sie diesen langen Leidensweg gehen musste, [...] das fand ich für sie fürchterlich, das fand ich für uns fürchterlich. Und ich glaube, wenn sie schneller gestorben wäre: Klar würde man sich dann genauso Vorwürfe machen, weil man sagt: hätte nicht sterben müssen. [...] Und wenn sie einen normalen Tod gestorben wäre, dann wäre es sicher anders gewesen, dann hätte ich mir keine Vorwürfe gemacht. “ (RY) Ein erschwertes Trauern kann somit sowohl durch den eigentlichen Verzicht ausgelöst werden, als auch durch die Gründe und Situationen, die zum FVET geführt haben. Besonders trat dies bei Teilnehmern auf, wenn sie den Sterbewunsch ihres Angehörigen nicht nachvollziehen konnten und an der Richtigkeit des Entschlusses zweifelten. Als weitere Ursache wurden die eigenen Werteinstellungen der Angehörigen identifiziert - lehnten diese den FVET als Freitod ihres Angehörigen ab, berichteten sie auch von einer erhöhten Belastung während und einer schwierigeren Trauerphase nach dem FVET. In der Erfahrung einiger Angehöriger war daher ein gemeinsames Gespräch im Vorfeld hilfreich.

Zwei Teilnehmer hatten die Erfahrung eines Suizids bei anderen Angehörigen machen müssen: Die Trauer nach einem FVET erlebten sie als dazu grundsätzlich unterschiedlich, was sie beim FVET mit der professionellen Begleitung und der Kommunikation mit dem Angehörigen verknüpften. ,Mein Vater hat sich das Leben genommen. Und damals gab es das andere noch nicht [erg. FVET mit Begleitung]. Und der musste ganz einsam in den Keller gehen, am Strick sich aufhängen. Und ich finde das einfach so traurig und ich finde so ein Palliativteam, so eine Betreuung, das ist ein Weg, den man gehen kann, und man hat [...] Hilfe von seinen Lieben. Und das ist ganz was anderes, als wie wenn ich jetzt sage: ,Du, ich gehe jetzt mal ... ‘ Mein Vater hat gesagt: ,Du ich gehe jetzt Zeitung holen. '- und kam nicht mehr. Also ich finde das einfach so traurig und das andere [erg. den assistierten Suizid in der Schweiz] finde ich auch keinen Weg, weil Wegfahren und dann alleine Zurückkommen - und irgendwann kommt der Sarg nach. Das ist alles keine Alternative. " (RE).

Zusammenfassend erschwert ein FVET nur in Ausnahmefällen den Trauerverlauf. Häufig liegt die Ursache dafür jedoch eher im Sterbewunsch als im Erleben des Verzichts selbst, insbesondere bei einem FVET als für die Angehörigen unverständlichem Freitod. Eine professionelle Begleitung wird in allen Phasen des FVET als Hilfe erlebt.

\section{Ethische Bewertung}

Im Folgenden sollen die qualitativen Erkenntnisse in die ethischen Überlegungen zum FVET und einer professionellen Begleitung eingebracht werden. Ohne ethisch normative Aussagekraft helfen sie jedoch bei der Identifizierung von relevanten ethischen Aspekten.

Drei Prämissen helfen, späteren Einwänden entgegenzutreten.

Erstens gelten die folgenden Überlegungen nur, wenn eine uneingeschränkte Einsichts- und Entscheidungsfähigkeit vorliegt, also kein Zweifel an der Authentizität der Entscheidung einer Person zum FVET besteht. Dies betrifft besonders die Ster- 
bewünsche, die als klare Willensäußerung, nicht als Teil einer Psychopathologie begriffen werden (Jaspers 1956, S. 302).

Zweitens soll von einer authentischen Beziehung zwischen Behandlungsteam und Patient ausgegangen werden - dass also eine Person ihre Motive zum FVET offenlegen und das Team sich im Einverständnis damit befinden kann. Die qualitativen Ergebnisse zeigen, dass dies nicht unbedingt für die rein physisch-somatische, dafür für die mit-menschlich zuwendende Beziehung zur begleiteten Person und den Angehörigen von Relevanz und im gemeinsamen Interesse ist. Strukturell ist dies wohl im Einverständnis zwischen Team und Patient gegeben (vgl. Jox et al. 2017).

Drittens wird als Prämisse gesetzt, dass die Zusage ärztlicher Begleitung eine Voraussetzung zur Realisierung eines FVET darstellt, wie für einen Teil der Personen (z.B. $X M$ ) gezeigt. Damit wird einer möglichen, aber nicht notwendig existenten Abhängigkeit der beiden Handlungen Rechnung getragen (McGee und Miller 2017). Ethisch wird die Bewertung der Handlung des FVET selbst nicht vollständig von der seiner professionellen Unterstützung getrennt: Käme man zum Ergebnis, dass der FVET ethisch unverantwortbar sei, wäre eine vorsätzliche ärztliche Unterstützung dabei ebenfalls abzulehnen.

Für die ethischen Überlegungen zum Freiwilligen Verzicht soll methodisch nach individualethischen und sozialethischen Argumenten unterschieden werden.

\section{Individualethische Bewertung des FVET und seiner medizinischen Begleitung}

Die gezeigten Ergebnisse der qualitativen Forschung lassen zwei Varianten von FVET nach dem Vorliegen einer terminalen Erkrankung unterscheiden. Für eine ethische Bewertung erscheint es sinnvoll, die beiden Fälle getrennt zu analysieren.

Der FVET bei Vorliegen einer terminalen Erkrankung ist ethisch zulässig und sollte palliativmedizinisch begleitet werden

Kritik am FVET selbst wurde besonders von verschiedenen christlichen Konfessionen in die Debatte eingebracht (Bormann 2019; Cavanagh 2014; Jansen 2004). Somit soll zur ethischen Analyse des FVET hier auf eine gemeinsame, klassische Ethiktradition zurückgegriffen werden, die des Thomas von Aquin.

Für die ethische Unterscheidung in verantwortbare oder nicht-verantwortbare Handlungen trennt Thomas in seiner Summa Theologiae (STh) drei Analysebegriffe voneinander ab, die als System so konstruiert sind, dass sie keiner weiteren Ergänzung bedürfen (Knauer 2002, S. 79f.). Zur qualitativen Bestimmung einer Handlung lassen sich der Gegenstand (finis operis) und die Absicht (finis operantis) an einer Handlung unterscheiden. Zur quantitativen Differenzierung wird das Modell um die Umstände (circumstantiae) ergänzt. Gegenstand und Absicht verhalten sich dabei etwa in Analogie zu den aristotelischen Begriffen Materie und Form (von Aquin 1888, STh I-II q18 a2 ad2-3). Mit dem Gegenstand, wörtlich übersetzt dem Handlungsziel, wird das bezeichnet, was die Handlung selbst darstellt, beispielsweise Brandstiftung oder das Spenden von Geld. Wie die beiden Beispiele leicht zeigen, hat der Gegenstand aus sich selbst oftmals schon einen ethischen Gehalt, kann aber auch ambivalent sein (von Aquin 1888, STh I-II q20 a1; Spaemann 2000). Er muss 
also um den Blickwinkel der Absicht, wörtlich das Ziel des Handelnden, ergänzt werden: Für Thomas unterscheidet sich die Handlung des selbstlosen Spendens von dem, das aus Eitelkeit oder Scheinheiligkeit geschieht (von Aquin 1888, STh I-II q20 a1). So können mittels der beiden Instrumente Handlungen qualitativ-ethisch beschrieben werde, die Umstände quantifizieren dann diese Handlungen, u. a. nach Ort, Zeit oder Ausmaß (von Aquin 1888, STh I-II q7 a1). Mit ihnen kann also z. B. zwischen dem Diebstahl eines Apfels vom Nachbarsbaum und einem Banküberfall unterschieden werden, wenn Gegenstand (Aneignung fremden Eigentums) und $A b$ sicht (persönliche Bereicherung) konstant sind (von Aquin 1888, STh I-II q7 a3 ad3).

In der Anwendung dieser Instrumente auf den FVET ist der Gegenstand der Handlung als solcher ambivalent. Denn ein freiwilliger Verzicht auf Essen und Trinken trägt aus sich selbst keinen ethischen Gehalt und muss von einer Absicht näher beschrieben werden, z. B. als religiöses Fasten. Beim FVET im Zuge einer terminalen Erkrankung legen die qualitativen Daten frei, dass die Absicht der Patienten vor allem der Wunsch nach Vermeidung von Leid und Schmerzen und nach einem Tod zu Hause ist (s. oben). ${ }^{6}$ Die Selbsttötung - der Hauptkritikpunkt in einem christlichen Koordinatensystem - bildet also nicht die wesentliche Absicht bei Personen mit terminalen Erkrankungen: Die Patienten haben zuvor meist alle kurativen Lösungen versucht und ausgeschöpft und würden sich, vor die Wahl gestellt, eher ein Leben ohne ihre Krankheit als den Tod selbst wünschen (vgl. FZ). ${ }^{7}$ Somit ist der FVET vor der Unausweichlichkeit einer zum Tode führenden Erkrankung als ethisch verantwortbar zu sehen. In der Praxis sollte er so eher wie das ethisch weithin akzeptierte Konzept und die palliativmedizinische Routine der Therapiezieländerung verstanden werden und damit als eine mögliche Option von Patienten am Lebensende bzw. mit einer erwartbar kurzen Lebenszeitprognose. Zuletzt offenbart Thomas von Aquins Modell ebenso die Unterschiede zu angesprochenen Sterbehilfevarian-

\footnotetext{
6 Zwar kennt Thomas an anderer Stelle die Unterscheidung von finis primarius und finis secondarius, also Primär- und Sekundärziel (von Aquin 1874, Super Sent lib4 d33 q1 a1 co). Als verkettete Absichten wäre das eigene Sterben als Sekundärziel zu denken, als Weg zur Erfüllung des finis primarius, ein Sterben zu Hause bzw. Leid- und Schmerzvermeidung. Dieser Einwand ist jedoch zu überdenken: Erstens muss beim Vorliegen einer terminalen Erkrankung vor der Unausweichlichkeit des nahen Lebensendes oftmals wohl zwischen dem Wunsch zu sterben und dem Wunsch schneller zu sterben differenziert werden. Zweitens wird bei Thomas ,unter Absicht gerade nicht das ,Ziel der Handlung“, sondern das ,Ziel des Handelnden“ verstanden“ (Spaemann 2000). Drittens zählt für die ethische Bewertung des finis für Thomas nur das größte, letztgestellte Ziel, aus dem die Handlung geschieht (von Aquin 1888, STh I-II q7 a4 ad2). Diese Einschätzung teilt auch Hörmann: „Weil sich also das Wollen hauptsächlich auf das Endziel richtet, wird es auch durch das Endziel am stärksten sittlich geprägt“ (Hörmann 1976, Sp. 1345-54).

7 Die Umstände des FVET spielen zuletzt eine untergeordnete Rolle: Zum Ausmaß des Verzichts illustriert Thomas zwar anhand von Essen und Trinken die Tugend des Maßes (temperantia) (von Aquin 1888, STh II-II q141 a6 ad2). Diese Tugend ist dort aber als relativ zu den Umständen zu begreifen - in den Extremfällen des Vorliegens einer terminalen Krankheit oder in der Sterbephase kann diese kaum gegen den FVET in Anschlag gebracht werden. Damit greift für diesen Fall auch der Begriff der Unterlassung (omissio) nicht (Birnbacher 2019), den Thomas in der Sündentheologie als Nicht-Erfüllung eines göttlichen Gebotes einführt: Eine Verfehlung durch omissio gilt nur für Handlungen, zu denen Menschen verpflichtet sind (Ricken 2017, S. 92f.). Letztlich hilft eine Abwägung nach Ort und Zeit, Konstellationen als ethisch unverantwortbar zu identifizieren, beispielweise dass ein Pilot einer Passagiermaschine zugleich einen FVET umsetzt. Für die palliativmedizinische Realität insbesondere von terminal Erkrankten bleibt dies glücklicherweise wenig relevant.
} 
ten: Ein FVET bei terminalen Erkrankungen ist so auch als ethisch verantwortbar zu sehen, da hier nicht eine primäre Selbsttötungs-Absicht vorliegt, noch ein ethisch umstrittener Gegenstand, wie eine Fremd- oder assistierte Tötung.

Kann der FVET bei terminalen Erkrankungen als ethisch verantwortbar eingestuft werden, gilt dies ebenso für seine professionelle Begleitung, zumal sie eine Hilfe für Patient und Angehörige darstellt. Patienten und ihren Angehörigen sollte diese, idealerweise durch ein auf ambulante Palliativversorgung zu Hause spezialisiertes Team (SAPV), unbedingt als Leistung angeboten und nach den Regeln ärztlicher Kunst zuteilwerden - immer vorausgesetzt, dass die Patienten dies wünschen.

\section{Der FVET als Freitod stellt eine Gewissensentscheidung für Patienten und ihr Behandlungsteam dar}

Eine Entscheidung für den FVET zum Freitod stellt die Absicht einer Selbsttötung in den Vordergrund. Damit hängt die ethische Bewertung und die der Begleitung zunächst von der philosophischen Bewertung des Suizids ab. Zu der aber besteht kein Konsens, lehnen mit jeweils eigenen Argumenten u. a. Platon, Aristoteles, Thomas von Aquin und Immanuel Kant den Suizid ab, während Seneca, David Hume, Friedrich Nietzsche oder Jean Améry ihn befürworten (Platon 1959, Phaidon 62b; Aristoteles 2007, NE 1138a; von Aquin 1888, STh II-II q64 a5; Kant 1900, MS, AA: VI, 442; Seneca 2007, epist. 70; Hume 2009, S. 7 ff.; Nietzsche 1968, S. 90; Améry 2008). Den Suizid als persönlich lebbares Problem fasst schließlich Albert Camus auf - und zugleich elegant zusammen: „Es gibt nur ein wirklich ernstes philosophisches Problem: den Selbstmord. Sich entscheiden, ob das Leben es wert ist, gelebt zu werden oder nicht, heißt auf die Grundfrage der Philosophie zu antworten“" (Camus 2000, S. 15). Somit erscheint es haltbar, zur individualethischen Frage der Zulässigkeit eines FVET als Freitod sich Karl Jaspers anzuschließen, der den Suizid in die Uneindeutigkeit entlässt, wo er zugleich ,inkommunikables Geheimnis des Einsamen“ (Jaspers 1956, S. 304) und „letzte Freiheit des Lebens“ (Jaspers 1956, S. 309) ist - und zur Gewissensentscheidung des Einzelnen wird.

Für die professionelle Begleitung nun folgt daraus:

Vertritt man die Position, dass ein Suizid prinzipiell zulässig und ein FVET in der freien Entscheidung einer Person begründet liegt, ist es folgerichtig, dass für eine Begleitung beim FVET das Gleiche wie beim FVET bei einer terminalen Krankheit gilt, also eine optimale, idealerweise SAPV-orientierte Betreuung des Patienten, wenn möglich zu Hause.

Gelangt man jedoch zu der Ansicht, dass ein FVET als Freitod abzulehnen ist, sprechen vor allem praktische Argumente dafür, diesen Patienten eine professionelle Begleitung dennoch nicht zu verweigern: Allem voran profitieren die Personen und ihre Angehörigen von dieser über alle drei Phasen des FVET hinweg: Bei der Entscheidungsfindung kann Hilfe angeboten und das Leid der Symptome gezielt gelindert werden, auch im Interesse der Angehörigen. Zudem wäre die Prämisse der vorliegenden Willens- und Entscheidungsfähigkeit mit der Anbindung an ein professionelles Team eher sichergestellt. Mögliche Fälle, wo ein FVET nicht als Freitod, sondern aus einer behandlungsbedürftigen psychischen Störung begonnen wird, könnten so leichter erkannt und Hilfe ermöglicht werden. 
Als Gewissensentscheidung ist sowohl die der sterbewilligen Person als die des Behandlungsteams zu achten. Personen des Behandlungsteams, die den Freitod weltanschaulich ablehnen, sollte die Begleitung eines solchen FVET somit prinzipiell freigestellt sein. Im Einzelfall werden diese Personen vor dem eigenen Gewissen zu prüfen haben, ob ihre Beteiligung nicht insgesamt eher bedeutet, Leiden zu verringern als einer Person den Weg zu erleichtern, den sie womöglich auch unabhängig beschreiten würde. Hier sei noch einmal an die vorsichtshalber gesetzte Prämisse erinnert, dass die Zusicherung professioneller Unterstützung den FVET ggfs. erst ermöglicht und FVET und seine Begleitung ethisch nicht vollständig getrennt werden können. Etwa in Anlehnung an die Freiheit, bei einem Schwangerschaftsabbruch im Normalfall nicht mitwirken zu müssen ( $\$ 12$ Schwangerschaftskonfliktgesetz), bedeutet eine Anerkennung als Gewissensentscheidung ethisch auch ein Nicht-Verpflichtungsgebot für die Begleitung beim FVET, sofern dieser als Freitod realisiert wird.

\section{Sozialethische Argumente}

Im letzten Teil soll die gesellschaftliche Tragweite einer medizinischen Begleitung beim FVET umrissen werden. Dabei kann nicht auf alle sozialethischen Überlegungen eingegangen werden. So werden hier drei typische Einwände gegen die Suizidhilfe auf den FVET bezogen (Jox 2013, S. $177 \mathrm{ff}$.).

\section{Hat der FVET negative Auswirkungen auf die Angehörigen und das Behandlungsteam?}

Aus Ländern, wo Personen ein assistierter Suizid möglich ist, legen empirische Ergebnisse nahe, dass auch hier das Vorliegen einer terminalen Erkrankung berücksichtigt werden sollte, wie es diese Arbeit tut: In Oregon, wo die Suizidhilfe nur ab einer Lebenserwartung von unter sechs Monaten zulässig ist (Death with Dignity Act, Oregon 1997, Oregon Public Health Division 2017), berichten Angehörige von keiner zusätzlichen psychischen Belastung (Ganzini et al. 2009). In der Schweiz gibt es bislang nur vereinzelt kantonal erlassene Gesetzesbestimmungen zum assistierten Suizid, sonst ist dieser jedoch erlaubt, sofern er nicht ,,aus selbstsüchtigen Beweggründen“ (Art. 115 Schweizerisches StGB) erfolgt. Hier zeigte sich eine erhöhte Prävalenz im Vergleich zur Normalbevölkerung von psychischen Erkrankungen (bes. hinsichtlich einer Posttraumatischen Belastungsstörung) bei Angehörigen von Personen, die diesen Weg wählten (Wagner et al. 2012). Beim FVET implizieren die Ergebnisse der eigenen Forschung einen ähnlichen Trend: Alle Teilnehmer, bei denen der Angehörige an einer terminalen Erkrankung litt, lehnten im Fragebogen die Aussage ab, der FVET habe ihr Trauern erschwert. Beim Freitod durch FVET jedoch berichteten nur einzelne Angehörige von einer erhöhten Belastung vor allem in der Entscheidungsphase, was sie mit ihrem Konflikt zum Freitod selbst und dem nicht nachvollziehbaren Sterbewunsch begründeten. Dies deckt sich mit den Ergebnissen vergleichbarer Forschung zum assistierten Suizid, wo die Entscheidung dazu meist nur im engsten Familienkreis diskutiert und dort von den Angehörigen zum Teil als Belastung erlebt wird (Gamondi et al. 2018). Im Fragebogen bewerteten die 
Angehörigen den FVET im Mittel als eine Belastung von 2,5 - wobei 0 keine und 10 eine extrem große Belastung bedeutete. Die kleine Fallzahl (18) lässt keine repräsentativen Aussagen zu, aber auch aus den Interviews heraus besteht kein Anhalt, dass der FVET hier prinzipiell einen stark negativen Einfluss hat. Wo eine ärztliche Begleitung des FVET gewährleistet war, erlebten alle Teilnehmer diese als Hilfe. Die Möglichkeit einer Begleitung sollte damit eher von Nutzen für die Angehörigen gesehen werden (Ivanovic et al. 2014).

In Hinblick auf das Behandlungsteam sei nochmals auf die vorgeschlagene Lösung eines Nicht-Verpflichtungsgebots hingewiesen, um negative Auswirkungen hier zu verhindern.

\section{Verändert sich durch den FVET das Berufsethos der Medizin?}

Die Freigabe einer medizinischen Suizidhilfe löst oft Befürchtungen aus, für Ärzte stelle das Leben eines Patienten dann womöglich nicht mehr den höchsten Wert dar (Jox 2013). Eine Begleitung, besonders beim Freitod-FVET, könnte hier also eine Nuance verschieben. Diese hat sich nur in der Palliativmedizin schon lange gefestigt, wenn es darum geht ,den Tagen mehr Leben, nicht dem Leben mehr Tage“ (C. Saunders) zu geben. Dieselbe Nuance vollzog sich im ärztlichen Blick auch in der Bekräftigung der Patientenautonomie nach Einführung der Patientenverfügung (Schicktanz et al. 2010). Für dieses ärztliche Ethos bezieht sich damit „das Zulassen des Todes eines Patienten [...] auch auf das Zulassen eines vorzeitigen und selbstverfügten Todes“ (Neitzke et al. 2013, S. 364). Die Begleitung des FVET vereint nun beide Aspekte: Ärztliche Hilfe erfolgt lediglich symptomorientiert und nach medizinischer Indikation, zugleich aber als Zulassen des vorzeitigen, selbstverfügten Todes. Eine Begleitung des FVET hätte so keine Veränderung des bestehenden Berufsethos zur Folge.

\section{Bringt der FVET mehr sozialen Druck auf Benachteiligte und eine schlechtere Palliativmedizin?}

Die Möglichkeit von Suizidhilfe könnte sozialen Druck auf gesellschaftlich benachteiligte, pflegebedürftige, behinderte oder auch nur alte Menschen ausüben, ihr kostenintensives - Leben nicht fortzusetzen (Klein 2018). Würde mit Ermöglichung der Begleitung des FVET also eine ,schiefe Ebene“ betreten, die langfristig nicht nur zur Annahme weiterer Formen der medizinischen Lebensbeendigung führen, sondern diese durch die normative Kraft des Faktischen auch zum gesellschaftlich akzeptierten Standard werden lassen und die Palliativmedizin eher zurückdrängen würden? (Jox 2013, S. 181 f.). Beim FVET scheint Letzteres nicht der Fall zu sein: Hohes palliativmedizinisches Können ist eine notwendige Voraussetzung für die Begleitung (Verhorst 2019; Wax et al. 2018).

Zudem liegt die „Tatherrschaft“ hier ganz beim Sterbewilligen: Die zum Tode führende Handlung ist keine medizinische noch notwendigerweise ärztlich unterstützte Handlung, womit konzeptuell eine klare Grenzziehung gegen eine ,schiefe Ebene" möglich wäre. 
Die Sorge vom Druck auf Benachteiligte hat sich in Oregon und den Niederlanden nicht bewahrheitet (Battin et al. 2007). Zudem setzt der FVET eine erhebliche Willensanstrengung voraus, was die Freiwilligkeit prozedural sicherstellen und eher gegen Missbrauch von Dritten verteidigen kann. Zuletzt wäre es entscheidend, bei der Freigabe der Begleitung des Freiwilligen Verzichts auf Nahrung und Flüssigkeit den ideellen Pflock einzuschlagen, dass sie nur vor der Autonomie des Patienten zu rechtfertigen ist (Menzel 2017). Mit Verweis auf diese Autonomie darf im Umkehrschluss dann von jedem, der sich nicht zum FVET entscheidet, auch das medizinisch und pflegerisch Mögliche zur Lebenserhaltung und -verlängerung unverändert eingefordert werden.

\section{Zusammenfassung}

Dieser Beitrag soll vor allem ein Beitrag zur anhaltenden Debatte um den Freiwilligen Verzicht auf Essen und Trinken sein: Zum einen indem er den Begriff des FVET konkretisiert als ein freies und in selbstbestimmter Entscheidung beschlossenes Unterlassen oder Verringern von Essen und Trinken im Bewusstsein, damit das eigene Lebensende herbeizuführen. Zum anderen möchte er vor den Ergebnissen eigener empirischer Forschung eine ethische Analyse und Bewertung des FVET starkmachen, die in der Unterscheidung zwischen medizinisch terminalen und nichtterminalen Situationen wurzelt und dabei individual- wie sozialethische Argumente gleichermaßen berücksichtigt.

Danksagung Mein erster Dank gilt allen Teilnehmerinnen und Teilnehmern für unsere Gespräche, ihre Offenheit darin und das damit geschenkte Vertrauen. Frau Dr. Beatrix Gerhard, Herrn Dr. Jürgen Bickhardt $(\dagger)$, Frau Dr. Johanna Anneser, Herrn Dr. Wolfgang Schweiger sowie Herrn Dr. Johannes Bükki danke ich für ihre Mithilfe beim Kontakt zu Angehörigen. In ganz besonderer Weise möchte ich mich bei Herrn Prof. Dr. Dr. Ralf Jox und Herrn Prof. Dr. Gian Domenico Borasio für ihre Betreuung des qualitativen Forschungsprojekts bedanken.

Funding Open Access funding provided by Projekt DEAL.

Open Access Dieser Artikel wird unter der Creative Commons Namensnennung 4.0 International Lizenz veröffentlicht, welche die Nutzung, Vervielfältigung, Bearbeitung, Verbreitung und Wiedergabe in jeglichem Medium und Format erlaubt, sofern Sie den/die ursprünglichen Autor(en) und die Quelle ordnungsgemäß nennen, einen Link zur Creative Commons Lizenz beifügen und angeben, ob Änderungen vorgenommen wurden.

Die in diesem Artikel enthaltenen Bilder und sonstiges Drittmaterial unterliegen ebenfalls der genannten Creative Commons Lizenz, sofern sich aus der Abbildungslegende nichts anderes ergibt. Sofern das betreffende Material nicht unter der genannten Creative Commons Lizenz steht und die betreffende Handlung nicht nach gesetzlichen Vorschriften erlaubt ist, ist für die oben aufgeführten Weiterverwendungen des Materials die Einwilligung des jeweiligen Rechteinhabers einzuholen.

Weitere Details zur Lizenz entnehmen Sie bitte der Lizenzinformation auf http://creativecommons.org/ licenses/by/4.0/deed.de.

\section{Einhaltung ethischer Richtlinien}

Interessenkonflikt P. Starke gibt an, dass kein Interessenkonflikt besteht. 
Ethische Standards Das Projekt inkl. der Befragungen wurde von der zuständigen Ethikkommission beraten (Aktenzeichen 18-761) und im Einklang mit nationalem Recht sowie gemäß der Deklaration von Helsinki von 1975 (in der aktuellen, überarbeiteten Fassung) durchgeführt. Von allen beteiligten Probanden liegt eine Einverständniserklärung vor.

\section{Literatur}

Améry J (2008) Hand an sich legen. Diskurs über den Freitod. Klett-Cotta, Stuttgart

von Aquin T (1874) Sancti Thomae Aquinatis commentum in quartum librum sententiarum magistri Petri Lombardi episcopi Parisiensis. In: Fretté SE (Hrsg) Doctoris angelici divi Thomae Aquinatis sacri ordinis F.F. praedicatorum opera omnia, Bd. 11. Vivès, Paris [super Sent.]

von Aquin T (1888) Summa theologiae. In: Sancti Thomae Aquinatis doctoris angelici opera omnia iussu Leonis XIII P.M. edita, cura et studio fratrum praedicatorum, Bd. 4-12, Rom [STh]

Aristoteles (2007) Die Nikomachische Ethik. Artemis und Winkler, München [NE]

Battin MP et al (2007) Legal physician-assisted dying in Oregon and the Netherlands: evidence concerning the impact on patients in "vulnerable" groups. J Med Ethics 33:591-597

Bernat JL et al (1993) Patient refusal of hydration and nutrition. An alternative to physician-assisted suicide or voluntary active euthanasia. Arch Intern Med 153:2723-2728

Bickhardt J, Hanke RM (2014) Freiwilliger Verzicht auf Nahrung und Flüssigkeit: Eine ganz eigene Handlungsweise. Dtsch Arztebl Int 111:590-592

Birnbacher D (2015) Ist Sterbefasten eine Form von Suizid? Ethik Med 27:315-324

Birnbacher D (2019) Freiwilliger Verzicht auf Nahrung und Flüssigkeit = ,passiver Suizid“ - was folgt? In: Coors M, Simon A, Alt-Epping B (Hrsg) Freiwilliger Verzicht auf Nahrung und Flüssigkeit. Medizinische und pflegerische Grundlagen - ethische und rechtliche Bewertungen. Kohlhammer, Stuttgart, S $106-119$

Birnbacher D, Walther C (2019) Selbstbestimmtes vorzeitiges Sterben durch Verzicht auf Nahrung und Flüssigkeit. PflegeRecht 4/2019:211-218

Bormann FJ (2019) Ein moraltheologischer Blick auf das sog. Sterbefasten. Z Med Ethik 65:261-280

Camus A (2000) Der Mythos des Sisyphos. Rowohlt, Reinbek bei Hamburg

Cavanagh M (2014) How should a Catholic hospice respond to patients who choose to voluntarily stop eating and drinking in order to hasten death? Linacre Q 81:279-285

Chabot B (2001) Sterfwerk. SUN, Nijmegen

Chabot B, Walther C (2015) Ausweg am Lebensende: Sterbefasten - Selbstbestimmtes Sterben durch freiwilligen Verzicht auf Essen und Trinken. Ernst Reinhard, München

Cicero (1998) Gespräche in Tusculum - Tusculanae Disputationes. Artemis und Winkler, München [Tusc.]

Coors M, Simon A, Alt-Epping B (2019) Freiwilliger Verzicht auf Nahrung und Flüssigkeit (FVNF): Ein einleitender Überblick. In: Coors M, Simon A, Alt-Epping B (Hrsg) Freiwilliger Verzicht auf Nahrung und Flüssigkeit. Medizinische und pflegerische Grundlagen - ethische und rechtliche Bewertungen. Kohlhammer, Stuttgart, S 7-12

Freiberger O, Kleine C (2015) Buddhismus: Handbuch und kritische Einführung. Vandenhoeck \& Ruprecht, Göttingen

Gamondi C et al (2018) Family caregivers' reflections on experiences of assisted suicide in Switzerland: a qualitative interview study. J Pain Symptom Manage 55:1085-1094

Ganzini L et al (2003) Nurses' experiences with hospice patients who refuse food and fluids to hasten death. N Engl J Med 349:359-365

Ganzini L et al (2009) Mental health outcomes of family members of Oregonians who request physician aid in dying. J Pain Symptom Manage 38:807-815

Hoekstra NL, Strack M, Simon A (2015) Bewertung des freiwilligen Verzichts auf Nahrung und Flüssigkeit durch palliativmedizinisch und hausärztlich tätige Ärztinnen und Ärzte - Ergebnisse einer empirischen Umfrage. Z Palliativmed 16:68-73

Hörmann K (Hrsg) (1976) Lexikon der christlichen Moral. Tyrolia, Innsbruck

Hume D (2009) Über den Freitod. In: Hume D, Kühn M (Hrsg) Über den Freitod und andere Essays. Beck, München

Ivanovic N, Buche D, Fringer A (2014) Voluntary stopping of eating and drinking at the end of life-a 'systematic search and review' giving insight into an option of hastening death in capacitated adults at the end of life. BMC Palliat Care 13:1

Jansen LA (2004) No safe harbor: the principle of complicity and the practice of voluntary stopping of eating and drinking. J Med Philos 29:61-74 
Jaspers K (1956) Philosophie. Band II - Existenzerhellung. Springer, Berlin

Jox R (2013) Sterben lassen. Über Entscheidungen am Lebensende. Rowohlt, Reinbek bei Hamburg

Jox R et al (2017) Voluntary stopping of eating and drinking: is medical support ethically justified? BMC Med 15:186

Kant I (1900) Gesammelte Schriften. Hrsg: Bd. 1-22 Preussische Akademie der Wissenschaften, Bd. 23 Deutsche Akademie der Wissenschaften zu Berlin, ab Bd. 24 Akademie der Wissenschaften zu Göttingen. Akademie-Ausgabe, Berlin [AA]

Kittelberger F (2018) Der Skandal liegt in der Verharmlosung durch die Verwendung des Begriffs „,Fasten“! Prax Palliat Care 41:21-24

Klein F (2018) Kommentar: Gefährlicher Ausweg Sterbefasten. Schmerzmedizin 34:10

Knauer P (2002) Handlungsnetze: Über das Grundprinzip der Ethik. Books on Demand, Frankfurt am Main

Mayring P (2015) Qualitative Inhaltsanalyse - Grundlagen und Techniken. Beltz, Weinheim

McGee A, Miller FG (2017) Advice and care for patients who die by voluntarily stopping eating and drinking is not assisted suicide. BMC Med 15:222

Menzel PT (2017) Voluntarily stopping eating and drinking: a normative comparison with refusing lifesaving treatment and advance directives. J Law Med Ethics 45:634-646

Neitzke G (2019) Grenzziehung zwischen Suizid und freiwilligem Nahrungsverzicht. In: Coors M, Simon A, Alt-Epping B (Hrsg) Freiwilliger Verzicht auf Nahrung und Flüssigkeit. Medizinische und pflegerische Grundlagen - ethische und rechtliche Bewertungen. Kohlhammer, Stuttgart, S 148-156

Neitzke G et al (2013) Empfehlungen zum Umgang mit dem Wunsch nach Suizidhilfe. Ethik Med 25:349-365

Nietzsche F (1968) Abt. 6, Bd. 1. Also sprach Zarathustra. In: Colli G, Montinari M (Hrsg) Nietzsche Werke. Kritische Gesamtausgabe. De Gruyter, Berlin

Oregon Public Health Division (2017) Oregon's Death with Dignity Act - 2017. https://www. oregonlegislature.gov/bills_laws/ors/ors127.html. Zugegriffen: 30. April 2019

Platon (1959) Phaidon. Ernst Heimeran, München

Quill TE et al (1997) Palliative options of last resort: a comparison of voluntarily stopping eating and drinking, terminal sedation, physician-assisted suicide, and voluntary active euthanasia. JAMA 278:2099-2104

Quill TE et al (2000) Palliative treatments of last resort: choosing the least harmful alternative. University of Pennsylvania Center for Bioethics Assisted Suicide Consensus Panel. Ann Intern Med 132:488-493

Radbruch et al (2019) Positionspapier der Deutschen Gesellschaft für Palliativmedizin zum Freiwilligen Verzicht auf Essen und Trinken. DGP. https://www.dgpalliativmedizin.de/phocadownload/ stellungnahmen/DGP_Positionspapier_Freiwilliger_Verzicht_auf_Essen_und_Trinken\%20.pdf. Zugegriffen: 26. Okt. 2019

Ricken F (2017) Thomas von Aquin über Sterben und Sterben lassen. In: Bormann FJ (Hrsg) Lebensbeendende Handlungen: Ethik, Medizin und Recht zur Grenze von ,Töten“ und ,Sterbenlassen`. De Gruyter, Berlin, S 87-93

Rosenau H (2016) §217 Strafgesetzbuch (StGB) - Neue Strafnorm gegen ein selbstbestimmtes Sterben in Deutschland. Bayr Ärztebl 70:100-102

Schicktanz S et al (2010) The cultural context of patient's autonomy and doctor's duty: passive euthanasia and advance directives in Germany and Israel. Med Health Care Philos 13:363-369

Seneca (2007) Epistulae morales ad Lucilium - Briefe an Lucilius. Artemis und Winkler, München [epist.] Spaemann R (2000) Einzelhandlungen. Z Philos Forsch 54:515-531

Verhorst A (2019) Zum pflegerischen Umgang bei Verzicht auf Nahrung und Flüssigkeit. In: Coors M, Simon A, Alt-Epping B (Hrsg) Freiwilliger Verzicht auf Nahrung und Flüssigkeit. Medizinische und pflegerische Grundlagen - ethische und rechtliche Bewertungen. Kohlhammer, Stuttgart, S 42-56

Wagner B et al (2012) Death by request in Switzerland: posttraumatic stress disorder and complicated grief after witnessing assisted suicide. Eur Psychiatry 27:542-546

Wax JW et al (2018) Voluntary stopping eating and drinking. J Am Geriatr Soc 66:441-445 